\title{
Estructuras de fábrica: enfoques «antiguos» y «modernos» para fenómenos muy locales
}

\section{Masonry structures: "old" and "new" approaches to strongly local phenomena}

\author{
$\underline{\text { F. Magdalena-Layos }}^{(*)}$, J. García-Muñoz ${ }^{(*)}$, D. Mencias-Carrizosa ${ }^{(* *)}$
}

\section{RESUMEN}

Para estudiar ciertos fenómenos de comportamiento local en las estructuras históricas de fábrica se propone la caracterización del material como un medio discontinuo. Se implementan tres métodos: uno experimental, fotoelástico, y dos numéricos, elementos finitos heterogéneos y bloques rígidos en contacto unilateral. De la comparación cualitativa de los resultados se deduce que las irregularidades aleatorias en el contacto entre piezas desempeñan un importante papel en la explicación del comportamiento local de la fábrica. La comparación cuantitativa con los resultados de ensayos de carga realizados previamente sobre muros aparejados en seco apoyan esta hipótesis. Los resultados obtenidos son muy esperanzadores y hacen pensar que es necesario profundizar en el desarrollo de enfoques de este tipo que, aún siendo relativamente comunes en el campo de los medios granulares, han sido muy escasos en el campo de las estructuras históricas de fábrica.

Palabras clave: Estructuras de fábrica; fotoelasticidad; elementos finitos heterogéneos; bloques rígidos; contacto unilateral.

\section{ABSTRACT}

The characterization of the material as a discontinuous media is proposed to study strongly local phenomena in the behaviour of historical masonry structures. Three methods are implemented: an experimental method, photoelastic, and two numerical methods, heterogeneous finite elements and rigid blocks in unilateral contact. Qualitative comparison of the results shows that the random irregularities in the contact between bodies play an important role in explaining local behaviour of masonry. Quantitative comparison with the results of load tests previously performed on dry masonry walls support this hypothesis. The results are very encouraging and suggest that it is necessary to develop approaches of this kind-approaches that, although relatively common in the field of granular media, have been very scarce in the field of historic masonry structures.

Keywords: Masonry structures; photoelasticity; heterogeneous finite elements; rigid blocks; unilateral contact.

${ }^{(*)}$ Escuela Técnica Superior de Edificación - Universidad Politécnica de Madrid (España).

${ }^{(* *)}$ Escuela Técnica Superior de Arquitectura - Universidad Politécnica de Madrid (España).

Persona de contacto/Corresponding author: fernando.magdalena@upm.es (F. Magdalena-Layos)

Cómo citar este artículo/Citation: Magdalena-Layos, F., García-Muñoz, J., Mencias-Carrizosa, D. (2016). Estructuras de fábrica: enfoques «antiguos» $\mathrm{y}$ «modernos» para fenómenos muy locales. Informes dela Construcción, 68(542): e150, doi: http://dx.doi.org/10.3989/ ic.16.032.

Licencia / License: Salvo indicación contraria, todos los contenidos de la edición electrónica de Informes de la Construcción se distribuyen bajo una licencia de uso y distribución Creative Commons Attribution License (CC BY) Spain 3.o. 


\section{INTRODUCCIÓN}

La evaluación de la seguridad de las estructuras históricas de fábrica presenta marcadas diferencias con respecto a la de otros tipos de estructuras. Existen numerosos métodos para el análisis del comportamiento global de este tipo de estructuras (1) (2), siendo cada uno de ellos, más o menos adecuado en función de las características del material, de los datos disponibles de éste y del fenómeno estudiado. Sin embargo, el análisis de los comportamientos locales se muestra bastante elusivo, especialmente cuando las propiedades e incluso la existencia del material de junta son dudosas y la resistencia del aparejo depende del rozamiento (3).

Una explicación en profundidad de las causas de esta dificultad excede el alcance de este trabajo, sin embargo, puesto que estos comportamientos pueden llegar a ser un problema práctico en determinados casos, es necesario proponer modelos alternativos que den una mejor aproximación a estos fenómenos locales.

Ensayos de carga realizados en el laboratorio de estructuras de la ETSAM-UPM (4), llevando a colapso por deslizamiento treinta y tres muros de mampostería en seco sometidos a la acción de cargas puntuales, han mostrado una distribución aleatoria y un amplio grado de dispersión en los resultados (Figura 1), que no puede explicarse por la dispersión en las propiedades de los materiales constituyentes.

Con el fin de investigar las posibles causas, se proponen tres métodos, uno de ellos experimental y los otros dos numéricos. El objetivo es contrastar la hipótesis de que la discontinuidad del medio y las inevitables irregularidades en el contacto entre partes desempeñan un papel relevante en la explicación de este tipo de comportamiento.
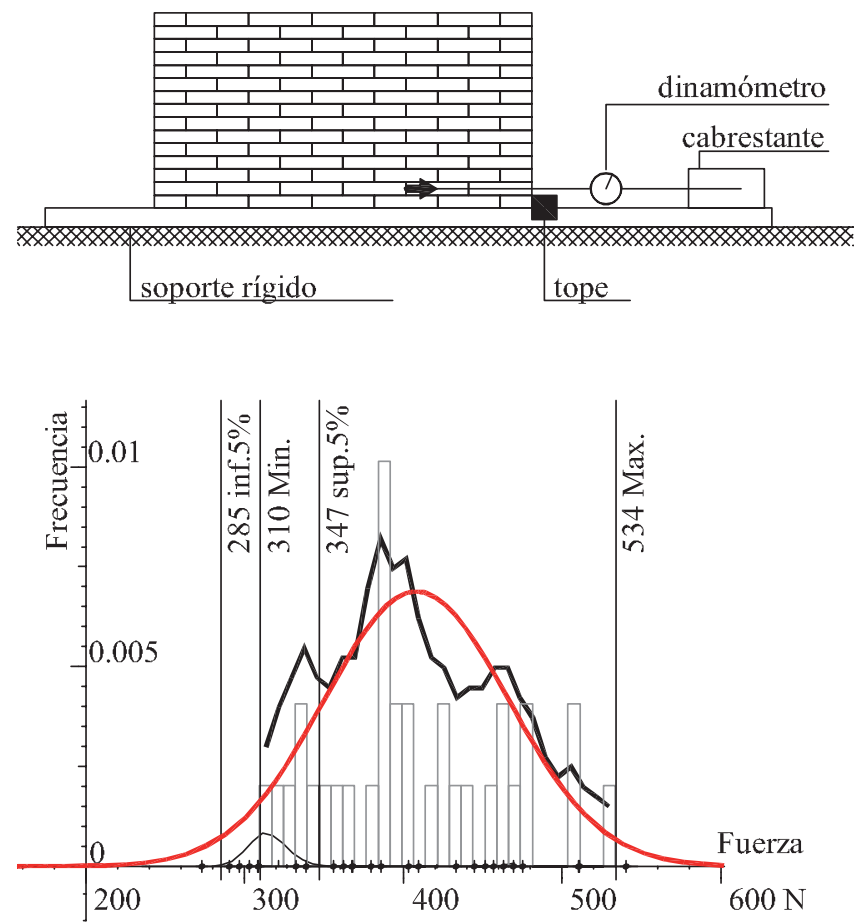

Figura 1. Esquema del ensayo de carga realizado en la ETSAMUPM y resultados obtenidos. En negro la gráfica de distribución de frecuencias de los resultados, en rojo la distribución gaussiana más ajustada, en números los estadísticos más significativos: máximo, mínimo e intervalo de confianza para el valor característico.
El trabajo se divide en dos partes. La primera se desarrolla sobre un modelo tomado de la literatura reciente, propuesto por Bigoni (5). Se trata de comparar los resultados de los tres métodos propuestos y comprobar si estos pueden dar indicios, a nivel cualitativo, de los motivos del comportamiento aleatorio. En la segunda se comparan los resultados de los ensayos de laboratorio con los de un modelo numérico, utilizando el menos costoso de los métodos numéricos propuestos.

\section{DESCRIPCIÓN DEL MODELO Y LOS MÉTODOS PROPUESTOS}

El modelo ensayado representa un muro de sillares aparejado en seco y compuesto por 187 piezas ortoédricas distribuidas en 22 hiladas. De ellas 165 son de proporción $2 \times 1$ y 22 de $3 \times 1$. Se disponen en un aparejo a soga convencional, formando un conjunto de $180 \mathrm{~mm}$ de ancho por 220 de altura. Se aplica una carga puntual vertical sobre el centro de la hilada superior utilizando una pieza de reparto auxiliar (Figura 2).

Se ha aplicado una carga de $100 \mathrm{~N}$ en el caso fotoelástico y $250 \mathrm{~N}$ en los análisis numéricos, con el fin de obtener una misma relación entre la carga aplicada y el peso propio, y por tanto similar dispersión horizontal de las tensiones de contacto, en los tres casos. La relación entre ambas cargas es la correspondiente a la de los productos entre el espesor y el peso específico en los diferentes modelos.

Se pretende analizar la irregularidad de la distribución de tensiones en los contactos entre caras mediante los siguientes tres métodos.

\subsection{Método fotoelástico}

La fotoelasticidad se emplea desde principios del siglo $\mathrm{xx}$ como método experimental para determinar las tensiones internas en sistemas estructurales (6).

El principio en el que se basan los ensayos fotoelásticos es simple: bajo luz polarizada, el fenómeno de la doble refrin-

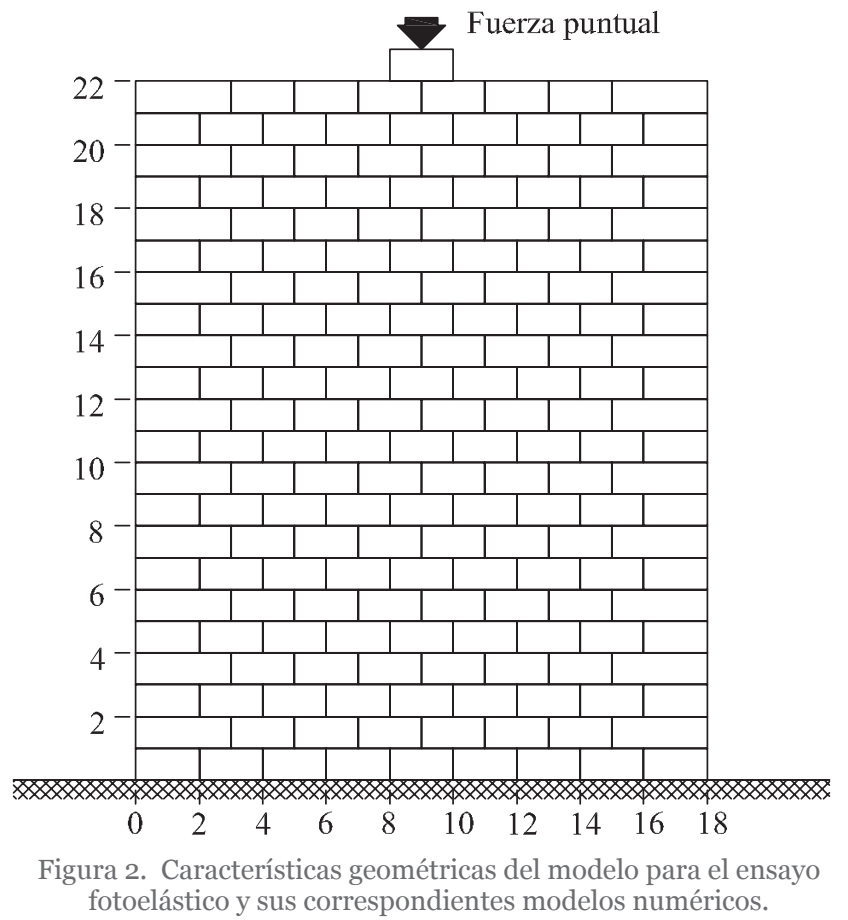


gencia en materiales traslúcidos se pone de manifiesto cuando estos se encuentran bajo tensión, lo que permite registrar estas manifestaciones de tensión con cualquier aparato de captación óptica. Los resultados que ofrece el método fotoelástico son así, de carácter indicial, lo que permite inferir, por vía de una contigüidad física directa, la presencia de fenómenos no detectables a simple vista. Estos resultados indiciales hacen posible establecer patrones de esfuerzo, determinar áreas de concentración de tensiones o comprender el comportamiento global del modelo. La cuantificación de valores tensionales, sin embargo, resulta difícil si se pretende obtener una precisión elevada, por lo que a estos ensayos suele otorgárseles un valor cualitativo.

Los ensayos fotoelásticos han sido habituales en el estudio de medios continuos (7), pero su empleo en el análisis de medios discontinuos es más reciente. Se ha usado con cierta asiduidad para el estudio de medios granulares (8), con frecuencia con la intención de evaluar el carácter aleatorio de las distribuciones internas de tensiones. Sin embargo, en lo que toca a los medios discontinuos de organización ortótropa, como las fábricas que se estudian en lo sucesivo, el uso de la fotoelasticidad ha sido muy restringido, y los intentos más sistemáticos y de cierto interés son sumamente recientes (5). No obstante, algunos hitos merecen reseñarse aquí.

McNicholas (9) empleó un revestimiento birrefringente para el análisis de las tensiones en un modelo de fábrica a escala 1:1. Heinrich (10) estudió un arco adovelado, con fuerte rozamiento en los lechos, y asoció tensiones internas en el material con líneas de empuje y mecanismos de colapso. Rajchenbach (11) propuso un estudio sobre un modelo de bloques rectangulares similar al aquí empleado, realizando un análisis de distribución vertical desde una óptica estadística. De forma reciente Bigoni (5) propuso un modelo de bloques rectangulares, que ensayó con diferentes materiales fotoelásticos y comparó con modelos matemáticos de distribución aleatoria. Por último Baig (12), empleando un modelo de igual geometría, diseñó un algoritmo de retoque infográfico para transformar la información fotoelástica en líneas o corrientes de tensiones.
Teniendo en cuenta estos antecedentes, se realizaron diversos ensayos sobre el modelo físico a pequeña escala de un muro de fábrica ya mencionado de Bigoni (5), disponiendo los bloques de resina en contacto seco y directo, asumiendo las irregularidades del corte. El material empleado en la fabricación de los bloques es una resina epoxídica de dos componentes, habitual en fotoelasticidad. Polimerizada a temperatura ambiente, su base es una variante comercial de la epiclorhidrina, y el agente endurecedor un monómero tipo BPA, mezclados en relación 3 a 1 en peso. El producto resultante es un sólido transparente, con matices amarillentos, para el que pueden estimarse una resistencias a compresión, tracción y flexión de $56,9,45,1$ y $55,90 \mathrm{~N} / \mathrm{mm}^{2}$ respectivamente, peso específico de valor $12 \mathrm{kN} / \mathrm{m}^{3}$ y un coeficiente de contracción lineal del $0,1 \%$. El material se ha producido por colada en molde de vidrio, hasta formar placas planas de $5 \mathrm{~mm}$ de espesor.

Éstas se han cortado después con una cortadora laser estándar (LC Universal XLS-10-MWH), combinando una alta potencia $(150 \mathrm{~W})$, para que el haz de laser atraviese la placa, con una velocidad media-baja del brazo $(0,01 \mathrm{~m} / \mathrm{s})$, para evitar el quemado de la resina.

Los bloques resultantes se han montado, en posición vertical, en una caja de confinamiento de PMMA (Figura 3). La caja se ha diseñado como un conjunto de placas y cercos que permita ensayar modelos planos. Las caras principales, que limitan frontalmente la tendencia al pandeo de los modelos, están separadas entre sí $6 \mathrm{~mm}$, de forma que entre el modelo, de $5 \mathrm{~mm}$ de espesor, y el confinador no exista contacto más que puntualmente, evitando así que el rozamiento entre ambos distorsione los resultados del ensayo. Los cercos perimetrales actúan, en la zona inferior, como base de apoyo del modelo $\mathrm{y}$, en los laterales, como elemento de contención de empujes cuando es necesario. Estrategias similares se han empleado en otros ensayos recientes (11).

Las caras anterior y posterior de la caja de confinamiento incorporan dos filtros polarizadores circulares de polivinilo alcohol-iodino, sin distorsión de color y de alta eficiencia de polarización (puede estimarse una transmisión cruzada de 0,002 a

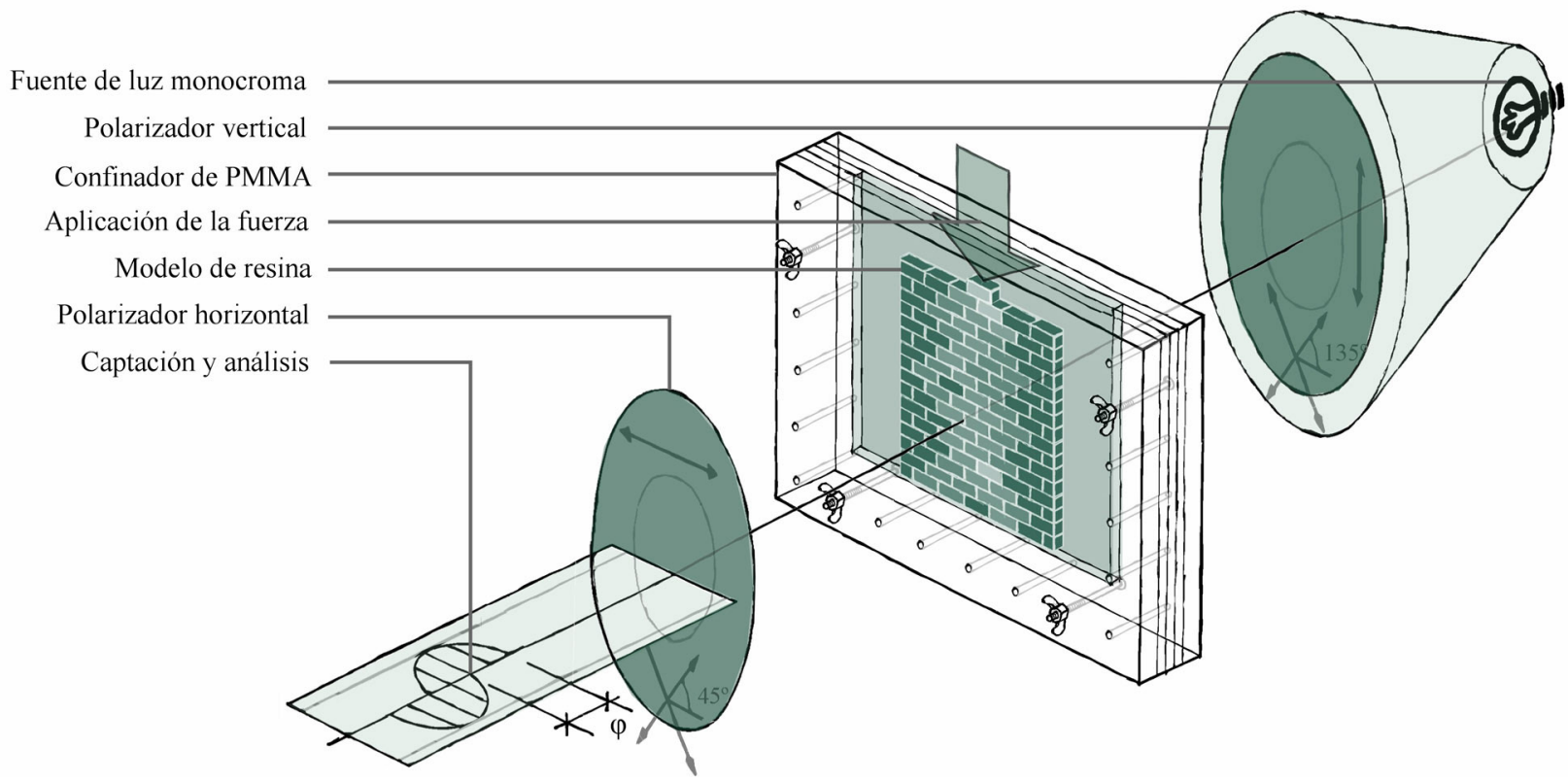

Figura 3. Condiciones de los ensayos fotoelásticos. 
$570 \mathrm{~nm})$. Cada filtro está compuesto por un polarizador lineal y una lámina de cuarto de onda al interior, una disposición que convierte al conjunto en un dispositivo similar a un polariscopio convencional, pero diseñado específicamente para ensayos a compresión de modelos de bloques. Para algunos detalles se emplearon, siguiendo el mismo esquema, sólo polarizadores lineales. La fuente de luz empleada es un emisor de diodo rojo, compuesto por 3 unidades LED de $5 \mathrm{~W} 10 \mathrm{COB} / 60^{\circ} \mathrm{RED}$, con un espectro emisor de $640 \mathrm{~nm}$, combinadas en un retroiluminador con reflector plateado y doble filtro difusor. El resultado es una fuente prácticamente monocroma, con una familia de curvas de luminosidad del diodo comprendidas entre los $600 \mathrm{y}$ los $680 \mathrm{~nm}$ de longitud de onda, muy concentradas en el entorno de los $640 \mathrm{~nm}$ desde los 10 hasta los $30 \mathrm{~mA}$, lo que permite realizar el ensayo sin monocromador (13).

La temperatura, registrada junto a las muestras, puede estimarse en los $21^{\circ} \mathrm{C}$ en superficie y en $22,5^{\circ} \mathrm{C}$ en ambiente. La carga se ha ejercido con el modelo en posición vertical, empleando un tornillo de presión acoplado a un eje fijo y diversas piezas interpuestas de PMMA, siendo el durmiente un bloque de resina de $20 \times 10 \times 5 \mathrm{~mm}$, y pudiendo estimarse una carga puntual aplicada sobre el durmiente de $100 \mathrm{~N}$, una tensión en las piezas más solicitadas de $0,5 \mathrm{~N} / \mathrm{mm}^{2}$ y una tensión media en la base de $0,1 \mathrm{~N} / \mathrm{mm}^{2}$. Se han realizado 16 ensayos de este tipo, reordenando los bloques de forma aleatoria. El registro fotográfico se ha realizado con una cámara Nikon D1oo, de $6 \mathrm{Mpx}$ efectivos, en tamaños de $3.008 \times 2.000 \mathrm{px}$, empleando lentes AF-S micro Nikkor (105 mm 1:2.8GED) y (70-180 mm 1:4.5-5.6D) para los detalles.

Los resultados obtenidos pueden leerse a simple vista, tanto a nivel global como a nivel local (Figura 4), siempre teniendo en cuenta que una mayor concentración de líneas isocromáticas (y no una mayor retroiluminación) implica una mayor tensión en el nudo (6).

Así, puede apreciarse como, a nivel global, los modelos manifiestan una campana con un ángulo de apertura semejante al que describen las simulaciones matemáticas de contraste. A nivel local, sin embargo, se aprecian de forma clara concentraciones irregulares en determinados puntos de contacto (Figura 5); concentraciones que generan discontinuidades, bien por exceso de solicitación, originando corrientes de tensiones, bien por defecto, dando origen a isletas de baja o nula tensión.
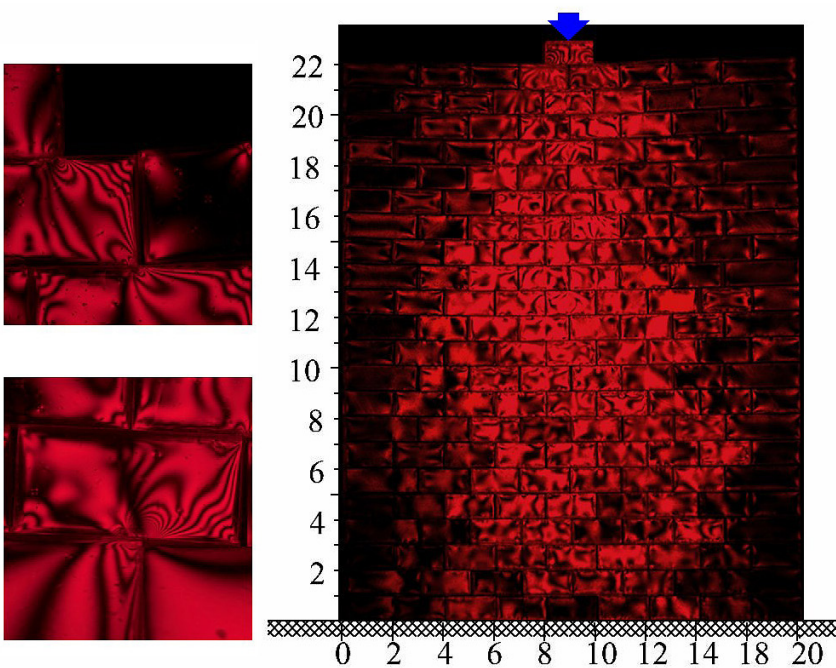

Figura 4. Resumen de resultados de los ensayos fotoelásticos.
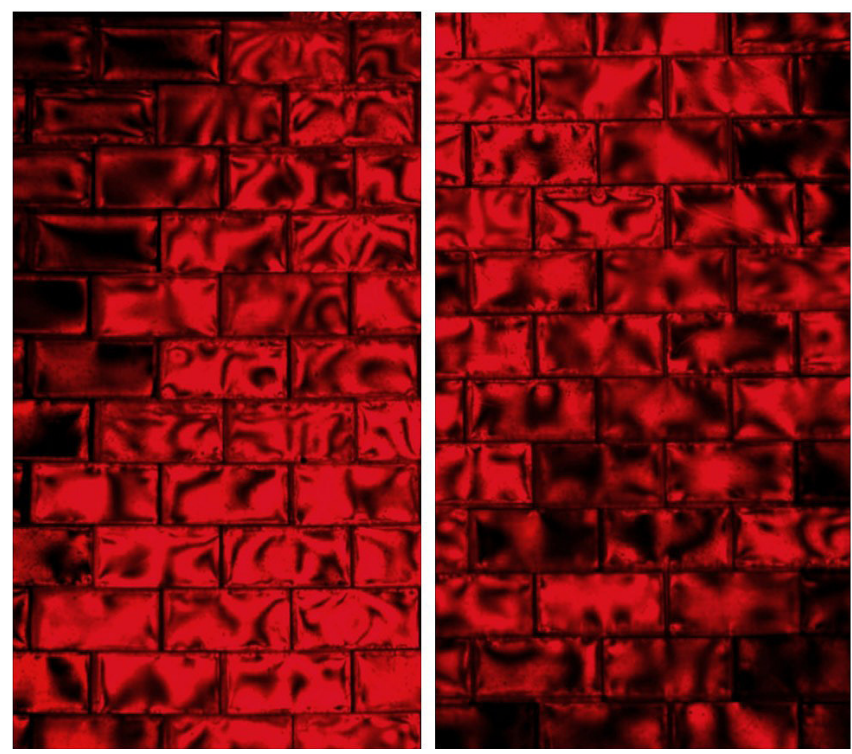

Figura 5. Corrientes de tensiones e isletas de baja tensión en uno de los modelos fotoelásticos ensayados.

\subsection{Método de elementos finitos heterogéneos}

El análisis elástico lineal mediante el método de los elementos finitos tiene por objeto determinar las tensiones verticales producidas por la carga exterior y así servir para localizar el «recorrido» de las cargas en el interior del muro de fábrica, empleando para ello el software SAP200o (14). Aunque no es un método muy extendido con esta finalidad, algunos autores (1), incluyen este procedimiento como enfoque avanzado para el análisis de estructuras históricas.

Al plantearse un acercamiento cualitativo, se pretende determinar las corrientes que recorre las cargas en el interior de la fábrica en lugar de precisar las tensiones de cada uno de los bloques o de describir las deformaciones, por lo que la caracterización del material no es determinante. Sin embargo, se han establecido los siguientes parámetros, correspondientes a fábricas, obtenidos a partir de los trabajos de Narayanan (15): Peso específico de valor, $15 \mathrm{kN} / \mathrm{m}^{3}$; Resistencia a compresión, $4 \mathrm{~N} / \mathrm{mm}^{2}$; Resistencia a tracción, o, $4 \mathrm{~N} / \mathrm{mm}^{2}$; Módulo de elasticidad, $4.000 \mathrm{~N} / \mathrm{mm}^{2}$ y Coeficiente de Poisson, 0,25 . Se utilizan elementos de $10 \mathrm{~mm}$ de espesor.

Para la realización de este modelo discontinuo con apoyo aleatorios entre piezas, el contacto entre las mismas se constituye mediante dos barras verticales siguiendo un patrón aleatorio de contacto, generado por un algoritmo informático. Este modelo es denominado por Baraldi (16) modelo de elementos finitos heterogéneos, añadiendo la particularidad del contacto entre piezas de manera aleatoria.

La carga aplicada tiene el valor de $250 \mathrm{~N}$ en el sentido negativo del eje vertical (Figura 2) para mantener la misma relación entre cargas exteriores y peso propio. Se realiza un análisis plano, anulando las fuerzas en el eje $\mathrm{Y}$, así como los giros en torno a los ejes Xy Z obteniendo las tensiones verticales S22 (Figura 6).

Con la intención de hacer un análisis cualitativo, no se han incluido los valores numéricos de tensiones pero sí los diagramas, que permiten conocer cómo se comporta el material. Para hacer más evidente y producir mayor contraste visual en la cuantificación de las tensiones se mostrado gráficamente 

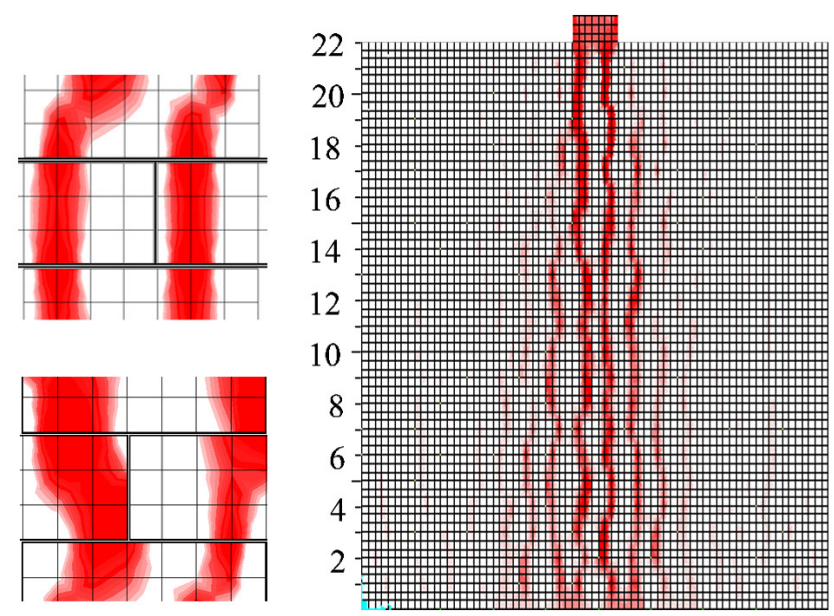

$\begin{array}{llllllllll}0 & 2 & 4 & 6 & 8 & 10 & 12 & 14 & 16 & 18\end{array}$

Figura 6. Distribución de tensiones verticales obtenida (derecha) y detalles de la misma (izquierda).

un rango determinado, con el fin de destacar las tensiones a lo largo del eje vertical de la fábrica y reducir la importancia de la concentración de tensiones bajo los puntos de aplicación de la misma, visualizando un rango de tensiones entre $\operatorname{los}-0,200 \mathrm{~N} / \mathrm{mm}^{2}$ y los $-0,046 \mathrm{~N} / \mathrm{mm}^{2}$.

\subsection{Método de bloques rígidos en contacto unilateral}

La carga, las características geométricas y las del material son las mismas que las del anterior modelo, excepto las correspondientes a la deformabilidad del material que en éste carecen de sentido. La presentación de este tipo de problemas mediante un conjunto de bloques en contacto, tiene una larga tradición en el análisis numérico de medios discontinuos, tanto en geomecánica (17) como en análisis estructural (18) (19). El método propuesto se encuadra entre los clasificados como «Advanced Computer Developments Based on Limit Analysis: Analysis of Blocky Structures» (1).

En su formulación mediante elementos discretos más elemental, el problema puede plantearse como uno de contacto unilateral entre bloques rígidos (20) (21), formularse matemáticamente como un problema de complementariedad lineal (22) y, al añadir como restricciones las condiciones de contacto aleatorias, resolverse mediante una secuencia de Programas Lineales. Para cada conjunto de condiciones de contacto se obtendrá una solución diferente. La utilización del procedimiento como núcleo de una simulación por el método de Montecarlo (23) permite hallar una muestra tan extensa como se desee de soluciones (24), una de las cuales se representa en la Figura 7.

La implementación para el caso en que sólo se consideran fuerzas y tensiones de contacto verticales es muy sencilla y consiste en:

1. Plantear las ecuaciones de equilibrio teniendo en cuenta que sólo se admiten tensiones de compresión en las superficies de contacto.

2. Elegir aleatoriamente los puntos de aplicación de las fuerzas de contacto.

3. Buscar una solución de equilibrio factible para la fuerza externa aplicada, utilizando Programación Lineal.

4. Si se encuentra, ésta será una de las posibles soluciones buscadas.
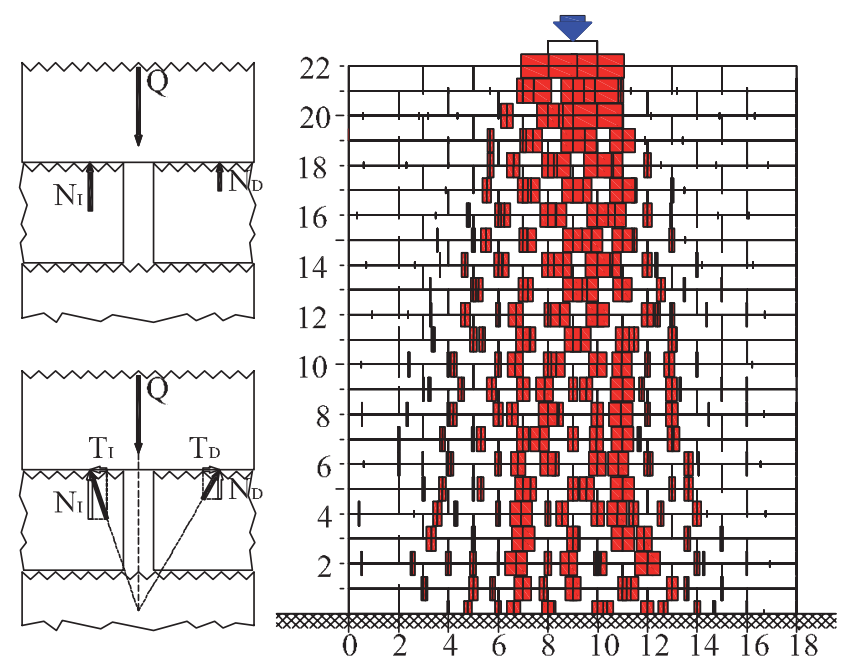

Figura 7. El elemento constituyente, sin (arriba) y con (abajo) tensiones tangenciales, y una de las soluciones.

Para poder comparar, a nivel cualitativo, con los resultados de los modelos anteriores se han representado los resultados gráficamente (Figura 7): a la izquierda el elemento básico constituyente del modelo, arriba sin tensiones tangenciales y abajo con ellas, y a la derecha un ejemplo representativo de los resultados. Los trazos en rojo, en proporción a su anchura, representan las tensiones de contacto.

\section{COMPARACIÓN CON LOS RESULTADOS DE LOS ENSAYOS DE CARGA}

Como segunda parte del trabajo se plantea una comprobación preliminar de que las hipótesis propuestas son plausibles también a nivel cuantitativo, para ello se ha desarrollado un modelo numérico correspondiente a los ensayos de carga. Se implementa una simulación por el método de Montecarlo utilizando el modelo de bloques rígidos en contacto unilateral y añadiendo a la formulación la ley rozamiento de Coulomb. Como valores de peso propio y coeficiente de rozamiento se toman los obtenidos en el ensayo. Dado que la fuerza de rozamiento en las caras de contacto horizontales está directamente relacionada con las fuerzas normales que actúan sobre ellas, puede entenderse como una extensión del método expuesto en 2.3 a todas las posibles líneas de rotura, siendo la principal diferencia en su implementación la sustitución de un Programa Lineal por una secuencia de Programas Lineales. Los fundamentos teóricos del método y los detalles de su implementación están expuestos en (25).

Se repite la simulación 1.000 veces con condiciones de contacto aleatorias y se tratan estadísticamente los resultados para compararlos con los del ensayo de carga.

En el gráfico de la Figura 8 se muestran los resultados de la comparación, en negro los resultados del ensayo y la distribución gaussiana (Normal) más ajustada, en rojo los resultados numéricos y su función de Densidad Estimada mediante Núcleos Gaussianos (26), también se señalan los intervalos de confianza (27) a un nivel del 99\% de los valores característicos -percentil del 5\%- de ambos. Se observa que el límite inferior para este valor característico está muy ajustado y que, en cualquier caso, la diferencia está del lado de la seguridad para el modelo numérico. 


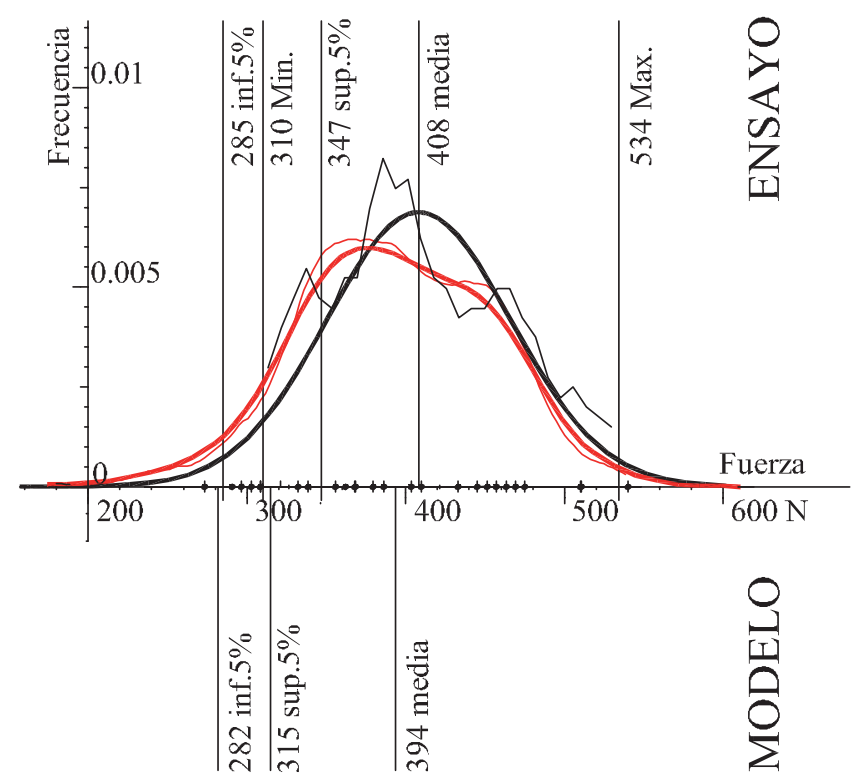

Figura 8. Comparación entre los resultados teóricos y experimentales.

\section{DISCUSIÓN}

El material constituyente de las estructuras de fábrica es heterogéneo, anisótropo, tiene una elevada resistencia a compresión pero escasa o nula resistencia a tracción, y es real o virtualmente discontinuo. Esta «discontinuidad» es de especial trascendencia en el estudio de comportamientos locales y, en cualquier caso, no considerar la aportación de la resistencia a tracción del material de la junta está del lado de la seguridad.

Un modelo de estas características es adecuado para un análisis simplificado de las construcciones históricas de fábrica, en las que no se dispone de un historial de los daños sufridos a lo largo del tiempo por dicho material y, en algunos casos, ni siquiera se tiene la seguridad de que éste exista en la actualidad.

Como medio para la obtención de indicios o evidencias experimentales del comportamiento interno de este material «discontinuo» se utiliza el método fotoelástico. Se ha dedica- do una mayor extensión a su exposición, porque siendo uno de los más antiguos para el análisis experimental de esfuerzos y bastante extendido en el estudio de medios discontinuos granulares (8), su uso no ha sido tan habitual en medios discontinuos ortótropos (5) (9) (11) (12). Además, en relación a la influencia de las irregularidades en el contacto entre elementos, el modelo fotoelástico no las predetermina, ya que éstas no son impuestas como una restricción sino producidas por el propio proceso de fabricación del modelo, tan exacto como ha sido posible utilizando corte por láser guiado por ordenador. En paralelo se han desarrollado dos modelos numéricos que intentan reproducir el comportamiento observado experimentalmente. En ambos, las posibles irregularidades en el contacto entre piezas se incorporan a través de la elección aleatoria de los puntos de paso para las resultantes de las tensiones de contacto.

En esta primera fase del trabajo se han obtenido resultados del ensayo de los modelos fotoelásticos, tanto continuo como discontinuo, y resultados cuantitativos para los dos modelos numéricos. Estos últimos se han representado gráficamente y en la Figura 9 se compara un ejemplo significativo de los resultados obtenidos con cada uno de ellos.

En todos los modelos discontinuos se puede observar la formación de «corrientes de tensiones» junto a otras zonas o «islotes» escasamente tensionados. La constatación de este fenómeno, bajo diferentes denominaciones como «cadenas de fuerzas» 0 «redes de tensiones», puede encontrarse en numerosos estudios sobre medios granulares, tanto numéricos como fotoelásticos. Este hecho tiene una importancia crucial para el estudio de comportamientos muy locales, como la acción de una carga puntual sobre un número pequeño de piezas.

Los tres métodos empleados hacen consideraciones muy diferentes respecto a la deformabilidad del material. En un extremo está el tercero que emplea cuerpos rígidos y en el opuesto la resina empleada que es un material bastante deformable, lo que parece, en alguna medida, influir en los resultados. Así, el ángulo de apertura obtenido entre la superficie de aplicación de la fuerza y la reacción en la base es algo mayor, en casi todos los casos ensayados del modelo fotoelástico, al obtenido en los diferentes modelos matemáticos, lo que tal vez sea atribuible a la mayor rigidez de estos últimos.

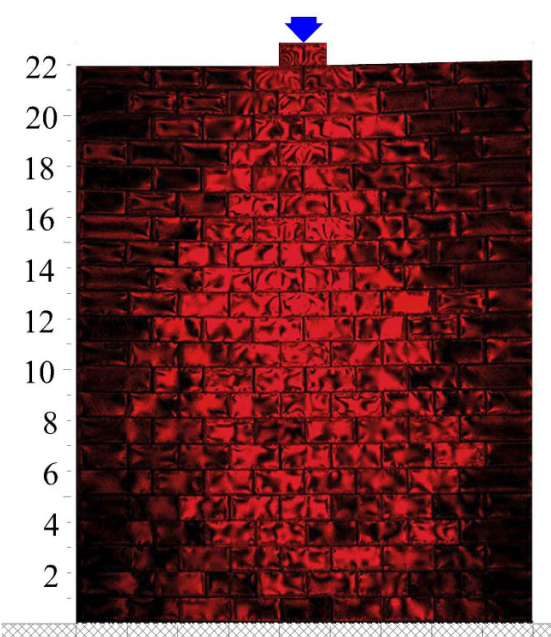

$\begin{array}{llllllllll}0 & 2 & 4 & 6 & 8 & 10 & 12 & 14 & 16 & 18\end{array}$

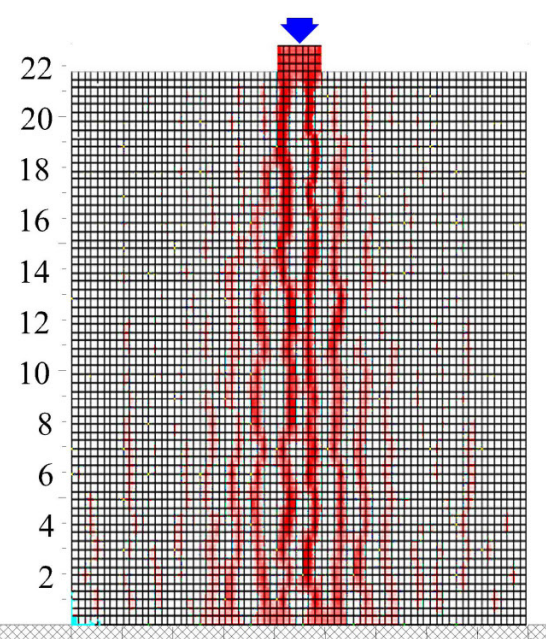

$\begin{array}{llllllllll}0 & 2 & 4 & 6 & 8 & 10 & 12 & 14 & 16 & 18\end{array}$

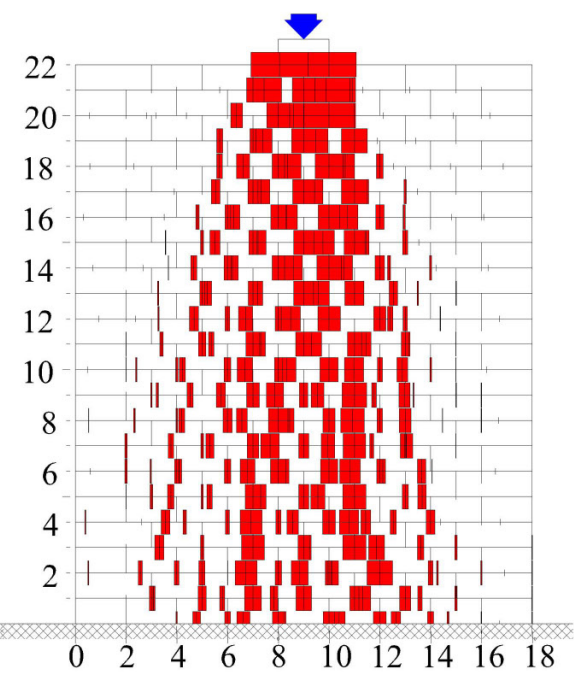

Figura 9. Comparación de los resultados obtenidos con los tres modelos: izquierda Fotoelástico, centro Elementos Finitos Heterogéneos, derecha Bloques Rígidos en Contacto Unilateral. 
Hay que aclarar que la intención de estos ensayos fotoelásticos ha sido la de comparar los resultados, de carácter indicial, con los de los modelos matemáticos y que, al contrario que en el trabajo citado de Bigoni, se ha utilizado un único tipo de material fotoelástico. Por tanto, aunque los resultados obtenidos son coherentes con los suyos, al emplear un único material el rango de respuestas ha sido más limitado. La comparación entre distintos materiales fotoelásticos para ver cual se adapta mejor al problema estudiado es un trabajo a desarrollar.

En lo respectivo a la comparación de la distribución de los resultados de los ensayos de carga realizados en la ETSAM-UPM con la de los resultados numéricos obtenidos mediante la simulación, el ajuste es bueno especialmente en el valor característico. En el conjunto de la distribución los resultados numéricos se mantienen consistentemente por debajo de los experimentales. Para comprobar si en el presente caso el uso del modelo puede considerarse seguro respecto de los resultados experimentales, se ha realizado un test de Kolmogorov-Smirnov para dos muestras (28) obteniéndose un altísimo p-valor de 0,994. Para un caso más general el ajuste será tanto mejor cuanto más se cumplan las hipótesis de partida, en especial la escasa o nula resistencia del material de junta, lo que correspondería en los estudios de medios granulares citados (11) a un material poco o nada cohesivo. La incorporación al modelo del material de junta, no tanto como elemento resistente sino como elemento de reparto, es un punto pendiente a desarrollar en futuros estudios.

Incluyendo tanto este último como la deformabilidad propia de los «bloques» se obtendrán sin duda resultados más ajustados a los obtenidos en los ensayos, aunque no más seguros. Habrá, por tanto, que sopesar cuidadosamente su incorporación mediante los coeficientes de seguridad adecuados, en función del grado de certidumbre que se tenga sobre ellos.

Aunque se deduce de lo dicho, conviene aclarar que todo esto no es aplicable al caso de fábricas modernas, en las que no existe el mismo grado de incertidumbre respecto a sus características.

\section{CONCLUSIONES}

La comparación entre los resultados obtenidos mediante los tres métodos empleados (Figura 9) permite apreciar grandes similitudes en lo que respecta a la aleatoriedad en la distribución de las tensiones de contacto. Aunque la influencia de la deformabilidad del material es un tema a estudiar en mayor profundidad, este comportamiento aleatorio se observa con claridad tanto en los diferentes ejemplos de los autores citados, realizados con materiales muy diversos, como en los tres métodos aquí presentados.

La distribución aleatoria de dichas tensiones produce efectos similares en todos los modelos: todos manifiestan, en dife- rente forma y grado, la aparición de corrientes de tensiones e isletas con tensiones muy bajas o nulas.

La comparación de los resultados obtenidos por la aplicación del segundo de los métodos numéricos con los del ensayo de carga sobre los muros de sillería en seco refuerza la idea de que la consideración tanto de la discontinuidad como de la aleatoriedad en las condiciones de contacto entre piezas desempeña un papel relevante en la explicación de su comportamiento resistente.

Aún admitiendo que el comportamiento global de una estructura antigua de fábrica puede no diferenciarse notablemente si se analiza como medio continuo o discontinuo, a nivel local sí se aprecian grandes diferencias si se considera un medio $\mathrm{u}$ otro, especialmente si se tiene en cuenta la incertidumbre en las condiciones de contacto entre piezas, como ocurre en muchos casos en las fábricas históricas. La aparición de islotes de tensiones muy bajas o nulas dentro de la fábrica, importante para el comportamiento a nivel local, pasa desapercibida cuando ésta se analiza globalmente.

De todo lo anteriormente expuesto puede inferirse que el tipo de aproximación propuesto, más habitual en los estudios sobre medios granulares (8), es -dentro de los límites aclarados en la discusión- pertinente en el análisis del comportamiento local de estructuras cuyo material pueda caracterizarse como un medio discontinuo.

A diferencia de los métodos basados en cálculo computacional, cuyos resultados dependen de las hipótesis de partida adoptadas, los métodos fotoelásticos -con las salvedades hechas respecto a la influencia del material- permiten un acercamiento más directo a los fenómenos estudiados permitiendo la visualización de los estados tensionales. En este contexto, la fotoelasticidad es un método útil para profundizar en el estudio del comportamiento local de las estructuras de fábrica. Sin embargo, dadas las limitaciones del procedimiento de análisis fotoelástico y la complejidad de la realización de ensayos de este tipo en número suficiente para permitir un análisis estadístico fiable, necesita del apoyo de otros métodos. Su empleo en combinación con los procedimientos de análisis numérico que aquí se proponen permite validar las hipótesis de partida en las que éstos se basan y hace posible un acercamiento práctico de gran interés a problemas locales, fundamentalmente en el caso de edificios históricos.

\section{AGRADECIMIENTOS}

El tercer autor quiere agradecer a la Fundación Juanelo Turriano, el apoyo a este trabajo por medio de la concesión de la beca para la realización de la tesis doctoral.

\section{REFERENCIAS}

(1) Roca, P., Cervera, M., Gariup, G. (2010). Structural analysis of masonry historical constructions. Classical and advanced approaches. Archives of Computational Methods in Engineering, 17(3): 299-325, doi: http://dx.doi.org/10.1007/ s11831-010-9046-1.

(2) Tralli, A., Alessandri, C., Milani, G. (2014). Computational methods for masonry vaults: a review of recent results. Open J. Civ. Eng., 8(1): 272-287, doi: http://dx.doi.org/10.2174/1874149501408010272.

(3) Magdalena, F. (2013). El problema del rozamiento en el análisis de estructuras de fábrica mediante modelos de sólidos rígidos. Tesis de doctorado. Universidad Politécnica de Madrid. 
(4) Magdalena, F., Aznar, A., Hernando, J. I., Magdalena, E. (2015). Sliding collapse in masonry structures: experimental tests. En CMMoST 2015 3rd International Conference on Mechanical Models in Structural Engineering, pp. 640-649. Sevilla, España.

(5) Bigoni, D., Noselli, G. (2010). Localized stress percolation through dry masonry walls. Part I-Experiments and Part II-Modelling. European Journal of Mechanics-A/Solids, 29(3): 291-307, doi: http://dx.doi.org/10.1016/j.euromechsol.2009.10.009.

(6) Frocht, M. M. (1965). Photoelasticity. London: J. Wiley and Sons.

(7) Dally, J. W., Riley, W. F. (1991). Experimental Stress Analysis, 3rd edition. McGraw-Hill Inc.

(8) Drescher, A., de Josseling de Jong, G. (1972). Photoelastic verification of a mechanical model for the flow of a granular material. J. Mech. Phys. Solids 20: 337-351, doi: http://dx.doi.org/10.1016/0022-5096(72)90029-4.

(9) McNicholas, J. B. (1970). Photoelastic Stress Analysis of Brick Masonry Systems. SIBMAC Proceedings. En 2nd International Brick and Block Masonry Conference, pp. 88-94. Stoke-on-Trent, England.

(10) Heinrich, B. (1977). Am Anfang war der Balken. Technik lernen mit übergreifenden Bezügen. Kultur \& Technik, 1: 38-45. Berlin: Deutsches Museum.

(11) Rajchenbach, J. (2001). Stress transmission through a cohesionless material. Mater. Phys. Mech., 3: 1-4.

(12) Baig, I., Ramesh, K., Hariprasad, M. P. (2015). Analysis of stress distribution in dry masonry walls using three fringe photoelasticity. En International Conference on Experimental Mechanics 2014, pp. 93022P-93022P. International Society for Optics and Photonics.

(13) Heywood, R. B. (1969). Photoelasticity for Designers, 1st edition. Pergamon Press.

(14) SAP2Ooo Advanced 14.o.o. Programa de análisis estructural. Computers and Structures, Inc. (2009).

(15) Narayanan, S. P., Sirajuddin, M. (2013). Properties of Brick Masonry for FE modeling. American Journal of Engineering Research (AJER). e-ISSN: 2320-0847.

(16) Baraldi, D., Cecchi, A., Tralli, A. (2015). Continuous and discrete models for masonry like material: A critical comparative study. European Journal of Mechanics - A/Solids, 50: 39-58, doi: http://dx.doi.org/10.1016/j.euromechsol.2014.10.007.

(17) Cundall, P. A. (1971). A computer model for simulating progressive large scale movements in blocky rock systems. En Proc. Symp. Rock Fracture (ISRM), vol. 1, paper II-8, Nancy.

(18) Livesley R. K. (1978). Limit analysis of structures formed from rigid blocks. International Journal for Numerical Methods in Engineering, 12(12): 1853-1871.

(19) Gilbert, M., Melbourne, C. (1994). Rigid-block analysis of masonry structures. Structural engineer, 72(21): 356-361.

(20) Fishwick, R. J. (1996). Limit analysis of rigid block structures. Tesis doctoral. Portsmouth: Department of Civil Engineering, University of Portsmouth.

(21) Ferris, M. C., Tin-Loi, F. (2001). Limit analysis of frictional block assemblies as a mathematical program with complementarity constraints. International Journal of Mechanical Sciences, 43(1): 209-224, doi: http://dx.doi.org/10.1016/ Soo20-7403(99)00111-3.

(22) Cottle, R. W., Pang, J. S., Stone, R. E. (2009). The linear complementarity problem. SIAM Classics in Applied Mathematics. doi: http://dx.doi.org/10.1137/1.978089871900o.

(23) Rubinstein, R. Y., Kroese, D. P. (2007). Simulation and the Monte Carlo method. Wiley.com. doi: http://dx.doi. org/10.1002/9780470230381.

(24) Magdalena-Layos, F., Hernando-García, J. (2014). Análisis límite de estructuras de fábrica como problema de contacto unilateral: un enfoque probabilista. Informes de la Construcción, 66(Extra-1): mo15, doi: http://dx.doi.org/10.3989/ ic.13.098.

(25) Magdalena, F., Hernando, J. I., Magdalena, E. (2015). Sliding collapse in masonry structures: a numerical model. En CMMoST 2015 3rd International Conference on Mechanical Models in Structural Engineering, pp. 629-639. Sevilla, España.

(26) Wand, M. P., Jones, M. C. (1995). Kernel Smoothing. London: Chapman \& Hall/CRC.

(27) Neyman, J. (1937). Outline of a Theory of Statistical Estimation Based on the Classical Theory of Probability. Philosophical Transactions of the Royal Society of London. Series A, Mathematical and Physical Sciences, 236(767): 333-380.

(28) Nikiforov A. M. (1994). Algorithm AS 288: Exact two-sample Smirnov test for arbitrary distributions. Appl. Stat., 43(1): 265-270. 